\title{
UNUSUAL POWER PRODUCTS AND THE IDEAL $\left[y^{2}\right]$
}

KATHLEEN B. O'KEEFE ${ }^{1}$

A power product $P=y_{i(1)} y_{i(2)} \cdots y_{i(n)}$, where $y_{i(j)}$ is the $i(j)$ th derivative of $y$, has weight $w=\sum_{j=1}^{n} i(j)$ and degree $d=n$. The sequence of integers $\left(e_{1}, e_{2}, \cdots, e_{n}\right)$, where $e_{k}=\sum_{j=1}^{k} i(k)-k(k-1)$, is called the weight sequence of $P$. According to a result of $\mathrm{H}$. Levi ([1], Theorem 1.2, p. 545), if some $e_{k}<0$, then $P \equiv 0\left[y^{2}\right]$. The discovery by D. G. Mead [2] that $Q=y_{1} y_{3} y_{4} y_{5} \equiv 0\left[y^{2}\right]$ shows that this criterion is not a necessary condition for membership in $\left[y^{2}\right] . Q$ is an unusual power product; that is, $Q$ is in $\left[y^{2}\right]$ and has a nonnegative weight sequence. By [3], there also exist unusual power products for $\left[y^{p}\right], p>2$; however, this note is concerned only with $\left[y^{2}\right]$. All unusual power products with weight sequences consisting of 0 's, 1 's, and 2's are described by Theorem IV, [2]:

A power product $P$ with weight sequence $\left(e_{1}, \cdots, e_{n}\right), 0 \leqq e_{i} \leqq 2$, $i=1, \cdots, n$, is unusual if and only if somewhere in the sequence at least one of the following patterns appears: $1,2,2,1 ; 1,2,2,2,2,0$; $0,2,2,2,2,1 ; 0,2,2,2,2,2,2,0$.

For particular arrangements of 0's, 1's, 2's, and 3's, similar results may be obtained; for example, using the notation of [2],

Theorem A. Let $g(a)=m\left(1,3_{1}, 3_{2}, \cdots, 3_{a}, 2,0\right),\left[3_{i}=3\right]$, then $g(a)=0$ if and only if $a=3$.

The purpose of this note is to show that, in general, a finite list of patterns will not suffice to describe unusual power products. By Theorem B there are unusual power products of arbitrarily high degree with no proper factors in $\left[y^{2}\right]$. The following results from [2] are stated for easy reference:

(a) $m(A, 0 ; B, 0)=m(A, 0) m(B, 0)$ for any sequences $A$ and $B$

(b) $m(1,0)=-2$

(c) $m(1,1, C)=-m(1, C)$ for any sequence $C$

(d) $m(1,2,1, D)=2 m(1, D)$ for any sequence $D$

(e) $m(0, E)=m(E)$ for any sequence $E$

(f) $m(1,2,2, F)=2 m(2, F)+m(1,2, F)$ for any sequence $F$.

Theorem B. Let $f(a)=m\left(1,2,3_{1}, 3_{2}, \cdots, 3_{a}, 1\right),\left[3_{i}=3\right]$; then $f(a)=0$ for all $a \geqq 1$. Furthermore, if $Q$ has the weight sequence $\left(1,2,3_{1}, \cdots, 3_{a}, 1\right), a \geqq 5$, then $Q$ has no proper factor in $\left[y^{2}\right]$.

Received by the editors May 17, 1965.

1 This paper was written while the author held a fellowship from the American Association of University Women. 
Proof. The theorem rcsts on two easy lemmas.

Lemma I. $m(1,2,3,3, S)=-m(1,2,3, S)$, for any sequence $S$.

Proof. $(1,2,3,3, S)$ is the weight sequence of $Q=y_{1} y_{3} y_{5} y_{6} \cdots$. Replacing $y_{5} y_{6}$ and using (a)-(d), (f), we get the equation

$$
m(Q)=-2 m(3, S)-2 \mathrm{~m}(1,3, S)-2 m(2,3, S)-m(1,2,3, S) .
$$

Use the equation $m(2,3, S)=-m(3, S)-m(1,3, S)$ to complete the proof.

In a similar fashion we prove

Lemma II. $m(1,2,3,2, S)=2 m(1,2, S)$, for any sequence $S$.

Returning to the proof of Theorem B, by Lemma I, $f(a)$ $=(-1)^{a-1} m(1,2,3,1)$. But $(1,2,3,1)$ represents the same power product as $(1,2,2,1)$, namely $R=y_{1} y_{3} y_{4} y_{5}$; and by Theorem IV of $[2], R \equiv 0\left[y^{2}\right]$.

If $P$ is a proper factor of $Q$, then $P$ is either an $\alpha$-term or a factor of

$$
\begin{aligned}
& P_{1}(k)=y_{1} y_{3} y_{5} y_{6} y_{8} \cdots y_{2 k}, \\
& P_{2}(k)=y_{1} y_{3} y_{4} y_{6} y_{8} \cdots y_{2 k}^{2}, \\
& P_{3}(k)=y_{1} y_{3} y_{5}^{2} y_{8} y_{10} \cdots y_{2 k}^{2}, \\
& P_{4}(k)=y_{0} y_{3} y_{5} y_{6} y_{8} \cdots y_{2 k}^{2}, \\
& P_{5}(k)=y_{1} y_{2} y_{5} y_{6} y_{8} \cdots y_{2 k}^{2} .
\end{aligned}
$$

$P_{1}(k)$ is comparable to the $\alpha$-term $y_{1} y_{3} y_{5}$ by Lemma I. $P_{2}(k)$ has the weight sequence $(1,2,2, \cdots, 2,0)$; hence, is in $\left[y^{2}\right]$ if and only if $k=4$. By Lemma II, $m\left(P_{3}(k)\right)=2 m\left(P_{2}(k-2)\right)$; and by (e) and (c) respectively, $m\left(P_{4}(k)\right)=m\left(P_{2}(k-1)\right)$ and $m\left(P_{5}(k)\right)=-m\left(P_{3}(k-1)\right)$. Thus $Q$ has no proper factor in $\left[y^{2}\right]$.

\section{BIBLIOGRAPHY}

1. H. Levi, On the structure of differential polynomials and on their theory of ideals, Trans. Amer. Math. Soc. 51 (1942), 532-568.

2. D. G. Mead, Differential ideals, Proc. Amer. Math. Soc. 6 (1955), 420-432.

3. K. B. O'Keefe, $A$ property of the differential ideal $\left[y^{p}\right]$, Trans. Amer. Math. Soc. 94 (1960), 483-497.

UNIVERSITY OF WASHINGTON 